Impact absorption properties of carbon fiber reinforced bucky sponges

This content has been downloaded from IOPscience. Please scroll down to see the full text.

Download details:

IP Address: 131.215.70.231

This content was downloaded on 03/04/2017 at 16:44

Manuscript version: Accepted Manuscript

Thevamaran et al

To cite this article before publication: Thevamaran et al, 2017, Nanotechnology, at press: https://doi.org/10.1088/1361-6528/aa6904

This Accepted Manuscript is: @ 2017 IOP Publishing Ltd

During the embargo period (the 12 month period from the publication of the Version of Record of this article), the Accepted Manuscript is fully protected by copyright and cannot be reused or reposted elsewhere.

As the Version of Record of this article is going to be / has been published on a subscription basis, this Accepted Manuscript is available for reuse under a CC BY-NC-ND 3.0 licence after a 12 month embargo period.

After the embargo period, everyone is permitted to use all or part of the original content in this article for non-commercial purposes, provided that they adhere to all the terms of the licence https://creativecommons.org/licences/by-nc-nd/3.0

Although reasonable endeavours have been taken to obtain all necessary permissions from third parties to include their copyrighted content within this article, their full citation and copyright line may not be present in this Accepted Manuscript version. Before using any content from this article, please refer to the Version of Record on IOPscience once published for full citation and copyright details, as permissions will likely be required. All third party content is fully copyright protected, unless specifically stated otherwise in the figure caption in the Version of Record.

When available, you can view the Version of Record for this article at: http://iopscience.iop.org/article/10.1088/1361-6528/aa6904 


\section{Impact absorption properties of carbon fiber reinforced bucky sponges}

Ramathasan Thevamaran ${ }^{1,2}$, Deepika Saini $^{3}$, Mehmet Karakaya ${ }^{3}$, Jingyi Zhu ${ }^{3}$, Ramakrishna Podila ${ }^{3}$, Apparao M. Rao ${ }^{3}$, Chiara Daraio ${ }^{1, *}$

${ }^{1}$ Division of Engineering and Applied Science, California Institute of Technology, Pasadena, CA 91125.

${ }^{2}$ Department of Materials Science and NanoEngineering, Rice University, Houston, TX 77005 .

${ }^{3}$ Department of Physics and Astronomy, Clemson Nanomaterials Institute, Clemson University, Clemson SC 29634.

*Corresponding author: daraio@caltech.edu

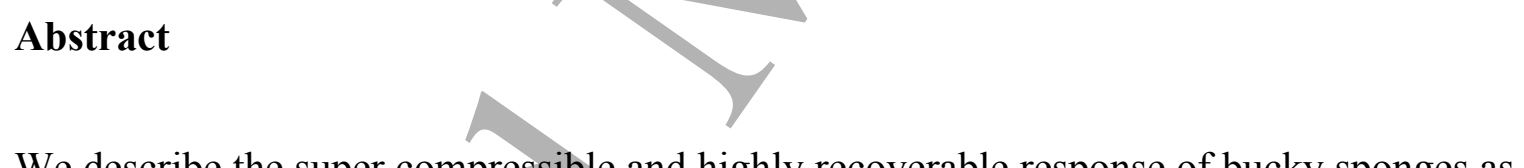

We describe the super compressible and highly recoverable response of bucky sponges as they are struck by a heavy flat-punch striker. The bucky sponges studied here are structurally stable, self-assembled mixtures of multiwalled carbon nanotubes (MWCNTs) and carbon fibers (CFs). We engineered the microstructure of the sponges by controlling their porosity using different CF contents. Their mechanical properties and energy dissipation characteristics during impact loading are presented as a function of their composition. The inclusion of CFs improves the impact force damping by up to $50 \%$ and the specific damping capacity by up to $7 \%$ compared to bucky sponges without CFs. The sponges also exhibit significantly better stress mitigation characteristics compared to vertically aligned carbon nanotube foams of similar densities. We show that delamination 
on the MWCNT-CF interfaces occurs during unloading, and arises from the heterogeneous fibrous microstructure of the bucky sponges.

Keywords: bucky sponges, heterogeneous materials, carbon fiber reinforced carbon nanotube composites, impact response, energy absorption, delamination.

\section{Introduction.}

Lightweight vibration isolating and impact absorbing materials are essential for a variety of applications such as controlling structural vibrations in automobiles and aircrafts [1], protecting spacecrafts from undetectable micrometeorite and space debris impacts [2], and absorbing shock in sport headgears $[3,4]$. Macroscale carbon nanotube (CNT)-based materials show high potential for protective applications because of their controlled physical, mechanical, and electrical properties, and their extremely low density [5-8]. Vertically aligned CNT foams have been synthesized with tailored microstructure and bulk density for applications requiring energy absorption and a broad range of mechanical properties [5,6,9-14]. Macrostructures of aligned CNTs with engineered shapes and geometries have also been synthesized as lightweight materials for efficient mechanical energy absorption $[15,16]$.

Random, self-supporting networks of CNTs - that are commonly referred to as CNT sponges $[17-21]$ - have been fabricated mainly for environmental applications such as sorption, filtration, and separation [17,22-25]. They are highly compressible up to $95 \%$ of their volume at low stress levels $(<0.25 \mathrm{MPa})$, have good fatigue resistance in response to repeated compressive cycles ( $\sim 100$ cycles), and present high strain recovery 
upon unloading (>90\%) [17]. When compressed, the intertwined CNTs in CNT sponges gradually form bundles and align in the direction perpendicular to the compressive loading direction [26], in contrast to the collective progressive buckling observed in the aligned CNT foams $[5,6]$. CNT sponges also exhibit viscoelastic response that is invariant over a broad range of temperatures from $-196{ }^{0} \mathrm{C}$ to $1000{ }^{0} \mathrm{C}$ [27]. The stick-slip motions of the interlocked CNTs in the sponges contribute to effective energy dissipation making them a potential protective material (damping ratio: 0.37-0.42) [27-29]. Layered structures consisting of alternating layers of non-aligned CNT sponges and aligned CNT arrays have also been created to obtain controlled deformations in desired locations $[11,26,30,31]$.

The mechanical responses of CNT sponges can be tailored significantly by coating the CNTs with different materials [32-34]. For example, coating the CNT sponges with a uniform conformal coating of 10-30 nm thick amorphous carbon has been shown to improve the elasticity and fatigue resistance, allowing the sponges to sustain $\sim 1000$ compression cycles without severe damage [33]. The presence of the coating, however, reduced the energy absorption during quasistatic cyclic loadings [33]. Coating CNT sponges with graphene has also been shown to improve their mechanical properties $[32,34]$. The presence of graphene overcoats on individual CNTs and at the nodal junctions of their network improved the Young's modulus of the CNT sponges by a factor of 6 [32], and increased the buckling load and energy absorption by a factor of 60 [34]. The fatigue resistance has also been improved by the graphene addition, where samples survived $\sim 2000$ cycles at $60 \%$ strain and $\sim 1$ million cycles at $2 \%$ strain without significant permanent damage [32]. 
Though there have been many studies in quasistatic loading regime, the dynamic behavior of bucky sponges, particularly concerning their ability to absorb impact, remains elusive. Here, we present the dynamic mechanical response and the energy absorption characteristics of self-assembled bucky sponges made of multiwalled CNTs and carbon fibers (CFs). We use an impact testing platform developed in our laboratory (Fig.1(a)) to characterize their dynamic behavior. We synthesized the bucky sponges using a scalable approach that can be adapted for industrial applications. We control their microstructure and porosity by selecting different weight percentages of CFs during synthesis. We have shown previously that bucky sponges exhibit a nonlinear foam-like stress-strain response, and have the ability to recover large strains up to $80 \%$ under quasistatic cyclic compression [23]. They dissipate energy through stress-strain hysteresis $\left(\sim 500 \mathrm{~kJ} / \mathrm{m}^{3}\right)$, which is $\sim 20$ times higher than the energy dissipative commercial polymeric foams of similar densities [23].

\section{Materials and methods.}

MWCNTs (NanoTechLabs, Inc.) having diameters between 30 and $50 \mathrm{~nm}$ were mixed with a surfactant ( $1 \%$ weight sodium dodecyl sulfate (SDS) aqueous solution) at $0.5 \mathrm{mg}$ $\mathrm{ml}^{-1}$. CFs having $\sim 8 \mu \mathrm{m}$ diameter were added to the solution in different proportions$10 \%, 20 \%$ and $50 \%$ by weight - to produce bucky sponges with three different microstructures. The prepared mixture was tip-sonicated using a Branson Sonifier (200 W) for 10-15 minutes at 40\% power, to homogeneously disperse the MWCNTs and CFs in the SDS solution. The solution was then vacuum filtrated and heat-treated in air at 70 ${ }^{0} \mathrm{C}$ for $30 \mathrm{~min}$. Finally, the dried samples were peeled off the filter membrane as standalone bucky sponges (Fig.1(b)). 

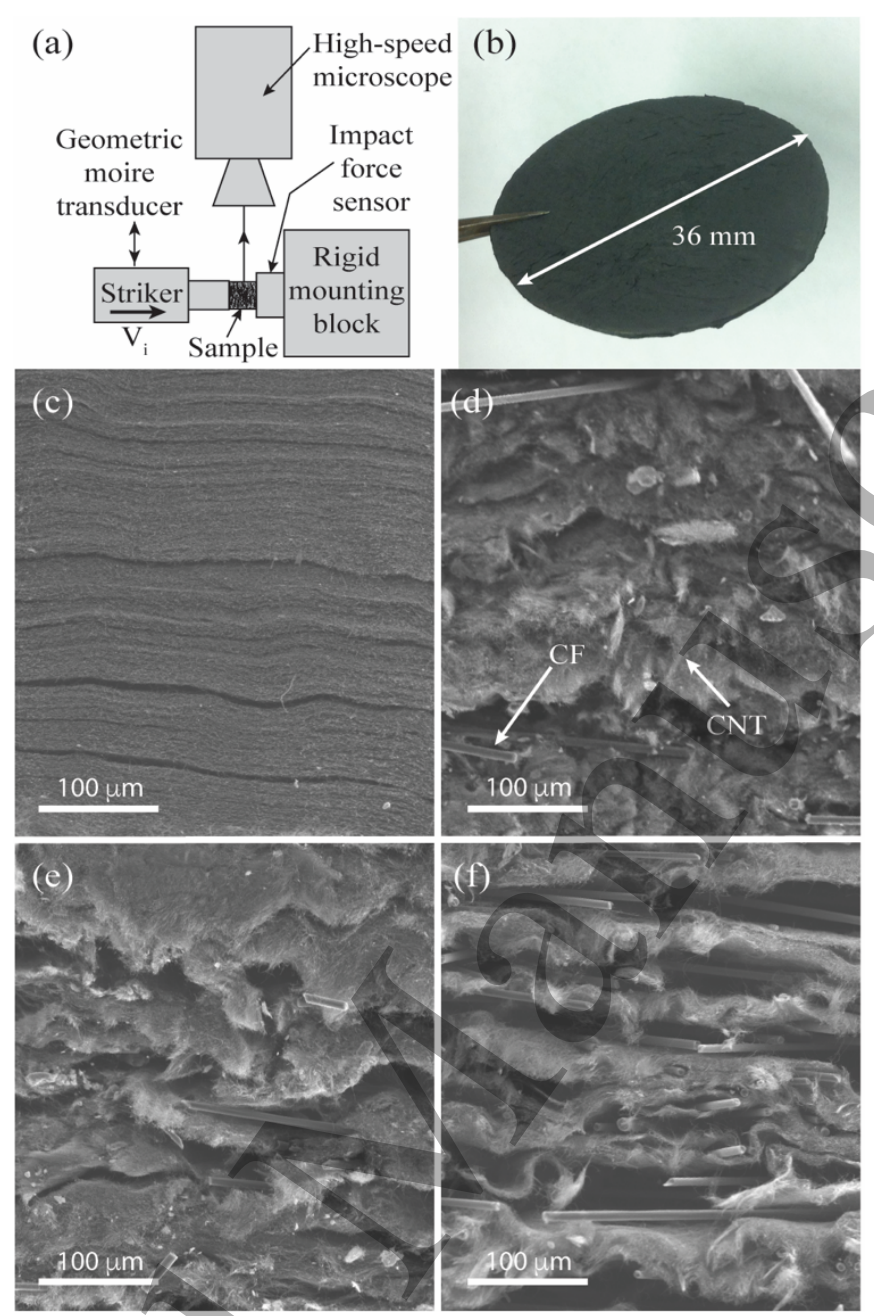

Figure 1. Dynamic characterization of bucky sponges: (a) schematic illustration of the dynamic compression testing setup, (b) pristine freestanding bucky sponge sample, (c-f) SEM images showing the microstructure of bucky sponge sample with (c) no CF inclusion $\left(\mathrm{CF}_{0}\right)$; (d) $10 \% \mathrm{CF}$ inclusion $\left(\mathrm{CF}_{10}\right)$, (e) $20 \% \mathrm{CF}$ inclusion $\left(\mathrm{CF}_{20}\right)$ and (f) $50 \%$ $\mathrm{CF}$ inclusion $\left(\mathrm{CF}_{50}\right)$.

These stand-alone material was then cut into 6.35 -mm-diameter-sized samples for dynamic testing using a custom-made core-drill. The synthesis process resulted in three different bucky sponges, denoted here as $\mathrm{CF}_{10}, \mathrm{CF}_{20}$ and $\mathrm{CF}_{50}$, where the number 
identifies the carbon fiber content by weight in bucky sponges $(10 \%, 20 \%$ and $50 \%$, respectively). An additional control sample was prepared with no carbon fiber inclusions (denoted as $\mathrm{CF}_{0}$ ). Scanning electron microscope (SEM) images in Figs. 1(c-f) show their microstructure. The $\mathrm{CF}_{10}$ samples have an average bulk density of $0.21 \pm 0.01 \mathrm{gcm}^{-3}, \mathrm{CF}_{20}$ of $0.20 \pm 0.01 \mathrm{gcm}^{-3}$ and $\mathrm{CF}_{50}$ of $0.15 \pm 0.01 \mathrm{gcm}^{-3}$, while the control samples $\left(\mathrm{CF}_{0}\right)$ have an average bulk density of $0.18 \pm 0.02 \mathrm{gcm}^{-3}$.

To determine the bucky sponge's mechanical response to impacts, we performed dynamic compression experiments on an impact testing setup built in our laboratory [35]. A simplified schematic showing the main components of the experimental setup is presented in Fig.1(a). The setup consists of an impact generator that delivers direct flatpunch striker impacts on stationary test samples, at controlled velocities between 0.5 to $10 \mathrm{~ms}^{-1}$. The striker $(7.08 \mathrm{~g})$ is nearly 500 times heavier than the sample, and delivers impacts at kinetic energies between 1 and $350 \mathrm{~mJ}$ in the impact velocity range we have tested. During the dynamic compression of the samples, a dynamic force sensor measures the transient force history, and a geometric moiré transducer measures the time-resolved dynamic displacements. A high-speed camera (Phantom V1610) with microscope lens (Infinity) was used for in-situ visualization and characterization of the microscale deformations. We also examined the samples in an SEM after impact, to identify the microstructural changes caused by the dynamic compression.

\section{Results and discussions.}

When a bucky sponge is impacted, the stress rises nonlinearly with strain up to a peak stress that corresponds to the maximum strain, and then declines rapidly as the striker is 
pushed back by the sample (Fig.2). The samples continue to recover as the striker unloads. Unloading path differs from the loading path forming a hysteresis that characterizes the energy dissipated during the loading-unloading cycle. Fig.2(a) shows the characteristic dynamic stress-strain responses of $\mathrm{CF}_{10}, \mathrm{CF}_{20}$ and $\mathrm{CF}_{50}$ sponges impacted at $4.55 \mathrm{~ms}^{-1}$. The increase in $\mathrm{CF}$ content from $10 \%$ to $20 \%$ shows stiffening in the stress-strain response (Fig.2(a)). The $\mathrm{CF}_{50}$ sponge, however, exhibited a more compliant response compared to the response of $\mathrm{CF}_{10}$ and $\mathrm{CF}_{20}$ sponges. Such compliant response arises from the higher porosity of $\mathrm{CF}_{50}$ sponges, which is evident in Fig.1(f). We performed similar impact tests, at five different velocities. The characteristic stress-strain responses of $\mathrm{CF}_{10}$ sponges for a range of impact velocities are shown in Fig.2(b). As expected, the deformation of the bucky sponges increases with increasing impact velocities, with compressibility reaching more than $70 \%$ of their height in the range of velocities tested. All samples exhibited high resilience to impact, by recovering more than $75 \%$ of their deformation upon unloading (Supplementary Fig.S1).
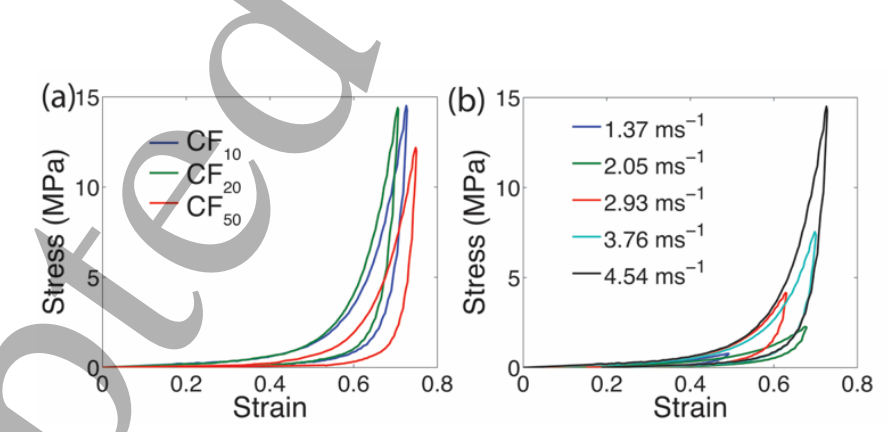

Figure 2. Dynamic stress-strain response of the CF-reinforced bucky sponges: (a) characteristic stress-strain responses of $\mathrm{CF}_{10}, \mathrm{CF}_{20}$ and $\mathrm{CF}_{50}$ samples, impacted at 4.55 $\mathrm{ms}^{-1}$, (b) characteristic stress-strain response of $\mathrm{CF}_{10}$ samples for a range of impact velocities. 
We compare the peak stress (Fig.3(a)) and the hysteretic energy dissipation-the area enclosed by the hysteresis loop (Fig.3(b)) — of the three different bucky sponges as a function of increasing impact velocities, as shown in Fig.3(a-b). Both of these parameters increase with impact velocity in all three bucky sponges. This is due to the increasingly higher maximum strains reached as the samples are impacted at increasing velocities. The $\mathrm{CF}_{50}$ sponges, however, damp the transmitted stresses more effectively than all other sponges (Fig.3(a)). This is a desirable characteristic for applications requiring impact stress attenuation. For example, the peak stress reached in a $\mathrm{CF}_{50}$ sponge at $4.5 \mathrm{~ms}^{-1}$ impact is $\sim 50 \%$ lower than the peak stress reached in a controlbucky sponge sample with no $\mathrm{CF}$ inclusions $\left(\mathrm{CF}_{0}\right)$, and $\sim 15 \%$ lower compared to $\mathrm{CF}_{10}$ and $\mathrm{CF}_{20}$ sponges (Supplementary Figure 2(a)). The hysteretic energy dissipation in all samples with CF inclusions is also reduced by $40-50 \%$ compared to control $\mathrm{CF}_{0}$ sponges (Supplementary Figure 2(b)). We attribute the reduction in hysteretic energy dissipation to the initial large deformations occurring at low-stress levels as the porous volume of the samples are compressed, and to the significantly/ reduced peak stresses compared to control $\mathrm{CF}_{0}$ sponges. However, the specific damping capacity - the ratio of hysteretic energy dissipated to the total energy absorbed up to the peak stress during loading-increases up to $7 \%$ with the inclusion of CFs compared to the control bucky sponges, $\mathrm{CF}_{0}$ (from 0.56 for $\mathrm{CF}_{0}$ to 0.60 for $\mathrm{CF}_{10}$ ). This suggests that the inclusion of $\mathrm{CF}$ induce the sponges to dissipate more energy/during the loading-unloading cycle. The energy that is not dissipated is stored elastically in the sample during loading and released back to the striker as the sample unloads. The elastic unloading modulus of the sample (Fig.3(c)) also increases with the impact velocity, due to the increasing densification of the sample under 
compression. The unloading modulus is calculated from the gradient of the unloading curve, corresponding to the first $5 \%$ of the unloading strain. These values are comparable for all three bucky sponges $\left(\mathrm{CF}_{10}, \mathrm{CF}_{20}, \mathrm{CF}_{50}\right)$ (Fig.3(a-c)). Among these three samples, the $\mathrm{CF}_{50}$ sponges exhibit slightly higher compliance (lower elastic modulus), lower peak stress, and lower energy dissipation, compared to $\mathrm{CF}_{10}$ and $\mathrm{CF}_{20}$ sponges, because of their highly porous microstructure (Fig.1(f)).
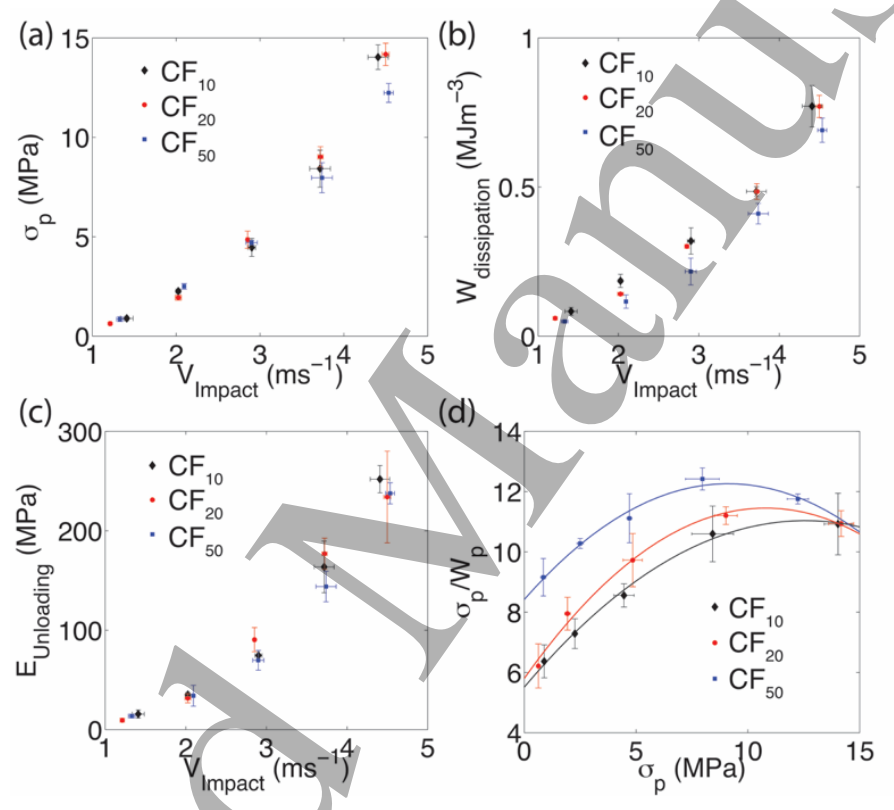

Figure 3. Dynamic properties of the CF-reinforced bucky sponges: (a) variation of peak stress with impact velocity; (b) variation of hysteretic energy dissipation with impact velocity; (c) variation of unloading modulus with impact velocity; (d) variation of dynamic cushion factor with peak stress. For clarity, data for the control sample is not included in this figure, but can be found in Supplementary Figure S2.

We characterize the cushioning ability of the bucky sponges from the dynamic cushion factor. We define the dynamic cushion factor $\left(\sigma_{\mathrm{p}} / \mathrm{W}_{\mathrm{p}}\right)$ as the ratio between the peak stress 
and the energy absorbed up to the peak stress, analogously to the definition of the quasistatic cushion factor [36]. The increase in energy absorption and/or decrease in peak stress results in low cushion factor, which is beneficial for protective applications. The variation of the dynamic cushion factor with peak stress is shown in Fig.3(d). It is evident that $\mathrm{CF}_{10}$ sponges exhibit better cushioning performance compared to $\mathrm{CF}_{20}$ and $\mathrm{CF}_{50}$ sponges. It should be noted that $\mathrm{CF}_{50}$ sponges, albeit having better ability for stress mitigation, exhibit lower cushion factor due to the lower energy absorption. The cushion factor vs. peak stress curves exhibit an unusual convex trend that is in contrast to the usual concave trend seen in quasistatic compression of foam-like materials [36]. This unique characteristic arises from the differences in the fundamental stress-strain response of the bucky sponges and of other foams [36]. Foam materials, in general, are characterized by an initial linear stress-strain response followed by a plateau regime at nearly constant stress level and finally, a densification regime with rapid increase in stress [36]. In such materials, increasing energy absorption (area under the stress-strain curve) at nearly constant stress level in the plateau regime leads to decreasing cushion factor. This decrease is followed by a rapidly increasing cushion factor in the densification regime where the peak stress increases rapidly. Consequently, typical curves relating the cushion factor to stress in foams show a concave trend [36], with a minimum corresponding to the best cushioning performance. In contrast, the bucky sponges exhibit nonlinear, monotonically increasing stress in strain with no apparent plateau regime. Due to this shape of the stress-strain curve, a competing effect arises between peak stress and the energy absorption that leads to the observed convex cushion factor curve in the tested impact velocity range. 
(a)
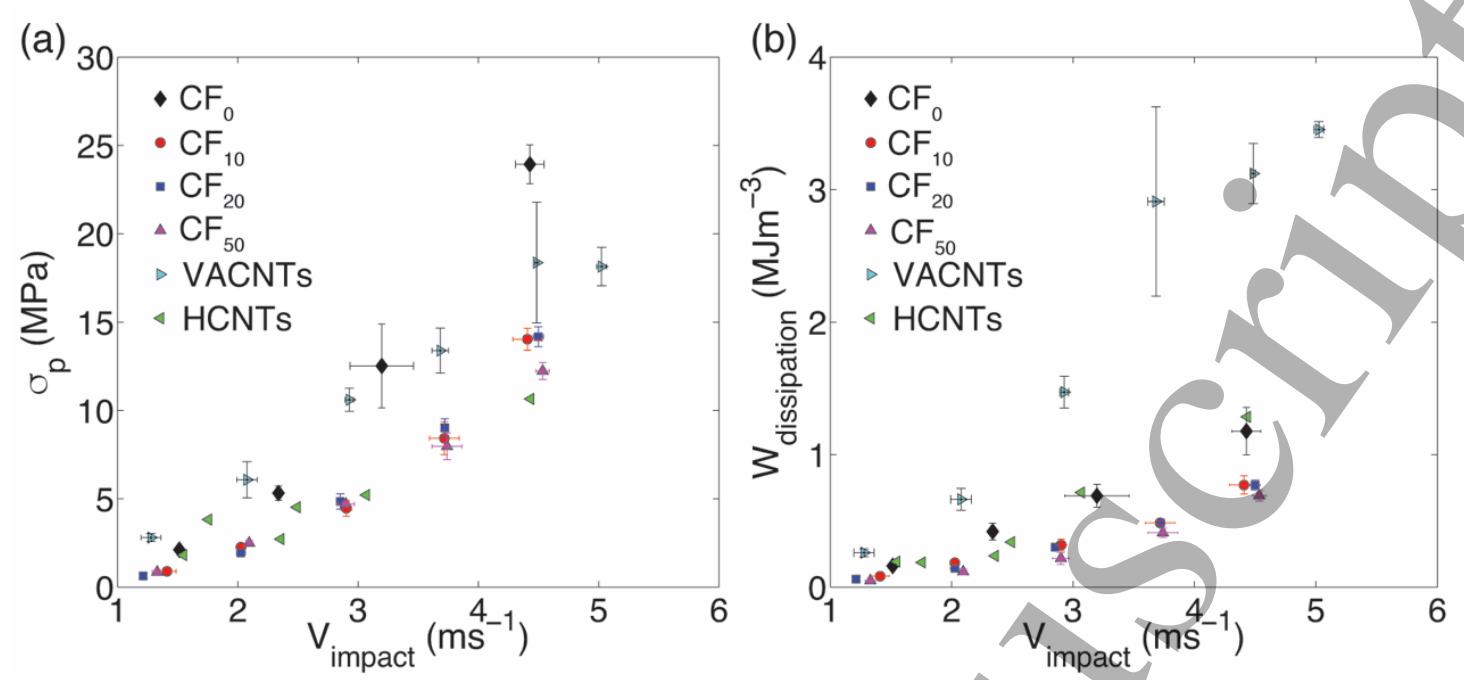

Figure 4. Comparison of (a) peak stress, and (b) energy dissipation of bucky sponges $\left(\mathrm{CF}_{0}, \mathrm{CF}_{10}, \mathrm{CF}_{20}, \mathrm{CF}_{50}\right.$; present study) with that of the vertically aligned CNT (VACNT) foams (previous study [6]), and helical CNT (HCNT) foams (previous study [37]).

We compare the dynamic response of bucky sponges with the dynamic responses reported previously for vertically aligned CNT (VACNT) foams [6], and helical CNT (HCNT) foams [37] in Fig.4. The bulk densities of the bucky sponges are comparable to that of the VACNT foams $\left(0.17 \pm 0.02 \mathrm{gcm}^{-3}\right)$ and HCNT foams $\left(0.15 \mathrm{gcm}^{-3}\right)$, and all samples were tested under similar conditions (same striker mass and similar impact velocities). The bucky sponges with $\mathrm{CF}$ inclusions $\left(\mathrm{CF}_{10}, \mathrm{CF}_{20}\right.$ and $\left.\mathrm{CF}_{50}\right)$ are more effective at reducing the transmitted stresses compared to VACNT foams or the control bucky sponges $\left(\mathrm{CF}_{0}\right)$, and are comparable to the response of HCNT foams (Fig.4(a)). The VACNT foams, however, have the ability to dissipate higher energy through hysteresis compared to the bucky sponges (Fig.4(b)). The formation and breaking of new van der Waals interactions during collective, progressive buckling of the VACNT foams $[5,6]$ lead to increased energy dissipation, and higher specific damping capacity (0.73) [6], 
which is $21 \%$ higher than the $\mathrm{CF}_{10}$ sponges. The larger hysteresis present in VACNT foams compared to bucky sponges is also evident from the dynamic stress-strain curves, where the VACNT foams reach higher peak stresses at lower maximum strains compared to bucky sponges. A set of characteristic stress-strain responses of bucky sponge samples and a VACNT foam sample impacted at similar velocities is shown in Supplementary Figure S3.
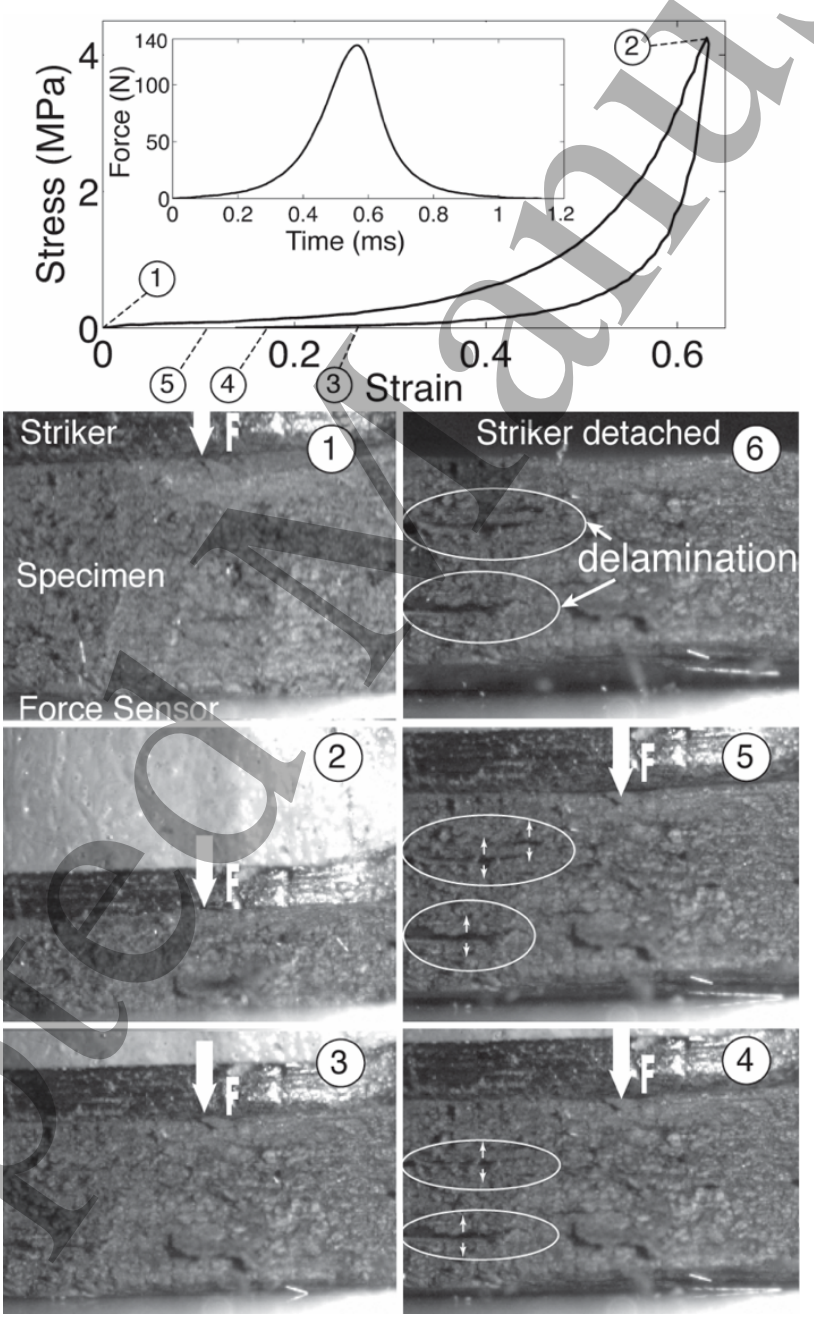

Figure 5. Dynamic response of a $\mathrm{CF}_{10}$ sponge impacted at $2.85 \mathrm{~ms}^{-1}$ striker velocity. The inset shows the corresponding force-time profile. Micrographs from the high-speed microscopy image sequence show the foam-like compression of the sample and the crack 
growth during unloading. The images (1-6) correspond to the indicated stress-states on the dynamic stress-strain diagram above.

We used high-speed microscopy for in-situ characterization of micro-scale deformation in bucky sponges. The high-speed microscopy revealed a uniform, foam-like compression of the bulk sponges under striker impacts (Supplementary video 1). This uniform deformation response, which is in contrast to the sequential progressive buckling observed in VACNT foams [5,6], has been reported previously in randomly aligned CNT networks as well [26]. The compressed samples recover instantaneously as the striker unloads, exhibiting high resilience to impact. The dynamic stress-strain and the forcetime responses of a $\mathrm{CF}_{10}$ sample (density: $0.21 \mathrm{gcm}^{-3}$, height: $1.83 \mathrm{~mm}$ ) impacted at 2.85 $\mathrm{ms}^{-1}$ are shown in Fig.5, along with a few snapshots from the high-speed image sequence that demonstrate the deformation mechanisms during loading and unloading. The sample underwent $\sim 60 \%$ compression and recovered more than $75 \%$ of its deformation. A closer look at the in-situ video reveals localized nucleation of microscale delaminations in several locations of the sample during unloading (Fig.5 micrographs (4-6), where the delaminations are highlighted by white circles). The nucleation of delamination is observable when the average stress on the sample decreases to very low stress levels, below $0.25 \mathrm{MPa}$, and the microscale delaminations continue to broaden as the bulk sample recovers further during unloading. Similar delaminations were observed in $\mathrm{CF}_{20}$ and $\mathrm{CF}_{50}$ samples as well. The occurrence of delaminations can be attributed to two main factors: (i) the intrinsic anisotropy in the alignment of the CNTs, and (ii) the presence of CFs, which makes the material heterogeneous. 

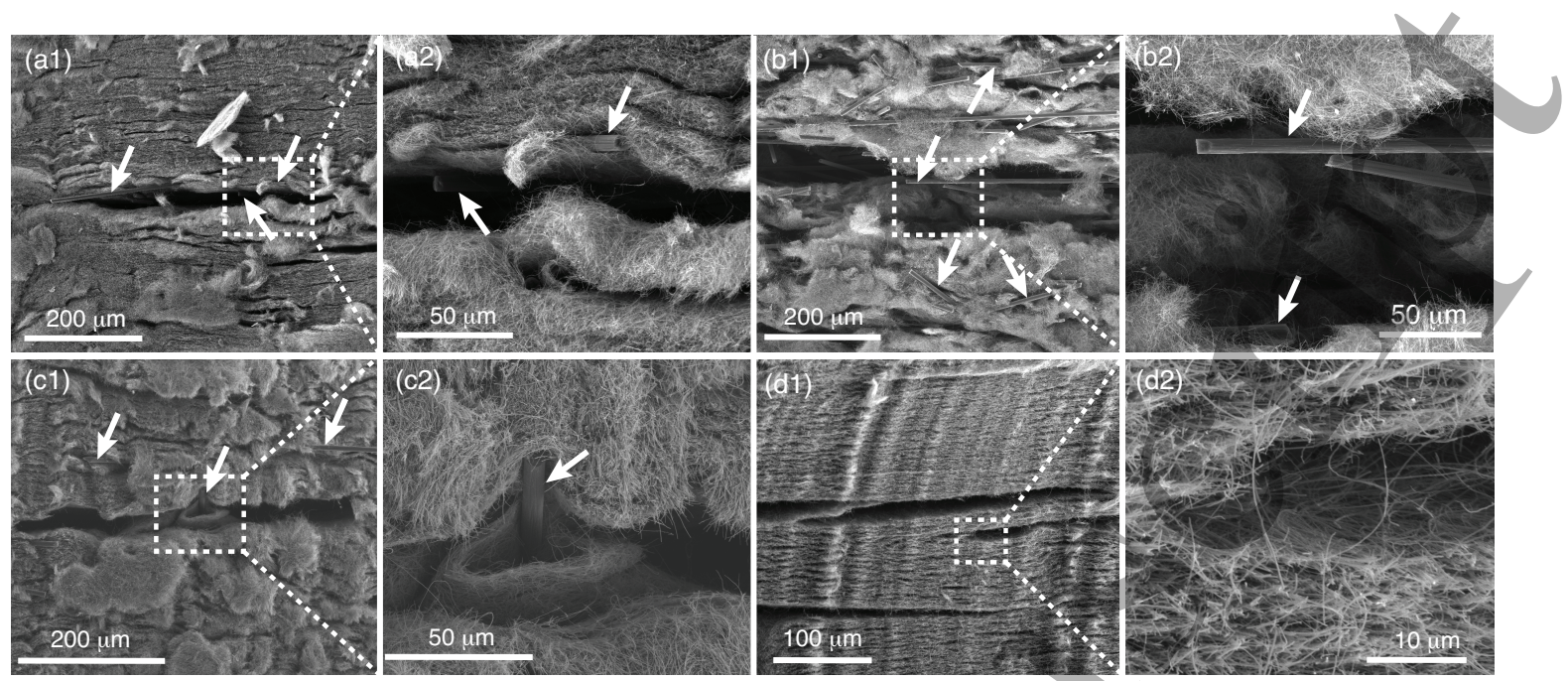

Figure 6. Post-impact SEM images showing the delamination cracks in the bucky sponges. (a1, a2) Cracks due to stress concentration around a $\mathrm{CF}$ in $\mathrm{CF}_{10}$ sponges. (b1, b2) Cracks formation due to increased presence of $\mathrm{CFs}$ in the $\mathrm{CF}_{50}$ sponges. (c1,c2) Cracks due to possible rotation of a stiff carbon fiber during compression in a $\mathrm{CF}_{10}$ sponge. (d1, d2) Cracks due to intrinsic heterogeneity in $\mathrm{CF}_{0}$ sponges. The arrows in the images indicate the locations of CFs in the sponges.

Post-impact SEM analysis of bucky sponges showed that the delamination occurred primarily in the areas where the CFs are present (Fig.6(a-c)). This suggest the effect of intrinsic lengthscale differences in CNTs and CFs. The largely different diameters of CNTs and CFs make the bucky sponges highly heterogeneous. When the sponges are compressed, the CNTs undergo more compaction in the vicinity of CFs, as the CFs act as stress concentrators. During unloading, such compacted regions cause local tensile stresses to develop in microscale, even though the bulk sample is under nominal compression at low-stress levels. We also observed rigid rotations or bending of some of the large CFs occurred during compression that could have caused delamination in their 
vicinity (Fig.6(c)). We also note that delaminations occur in the direction that is normal to the loading direction, because of the described microscale deformation modes, nonuniform stress distributions, and the inherently anisotropic self-assembly of CFs inside the CNT network during synthesis.

We observed similar delaminations in the control bucky sponge samples with no carbon fiber inclusions $\left(\mathrm{CF}_{0}\right.$, Fig.6(d)). However, in the case of $\mathrm{CF}_{0}$ sponges, the delamination is mainly due to the intrinsic anisotropy of the material arising from the synthesis processmixing, filtering and vacuum compaction. Even though CNTs are randomly oriented during mixing, they self-assemble into layers during vacuum filtration due to their large aspect ratio — as it is evident from the SEM images of Fig.1(c) and Fig.6(d).

\section{Conclusions}

We synthesized bucky sponge samples with different microstructures using a synthesis approach that is potentially scalable for large-scale industrial applications. Using controlled striker impact testing, we characterized their dynamic response and energy dissipation characteristics as a function of impact velocity and composition. The inclusion of CFs improves the ability of bucky sponges to mitigate impact stresses. In addition to their unique cushioning characteristics, their intrinsic heterogeneity and the microscale deformation modes lead to delamination during unloading. These findings provide insights into the fundamental deformation mechanisms of sponge materials with heterogeneous fibrous microstructure. Bucky sponges can find applications in the development of impact protective and structural vibration damping materials due to their energy absorption characteristics and ease of fabrication. 


\section{Acknowledgements}

We acknowledge financial support from the Institute for Collaborative Biotechnologies (ICB) under the contract W911NF-09-D-0001 with the Army Research Office (ARO).

\section{References}

[1] Rao M D 2003 Recent applications of viscoelastic damping for noise control in automobiles and commercial airplanes J. Sound Vib. 262 457-74

[2] Grossman E, Gouzman I and Verker R 2010 Debris/micrometeoroid impacts and synergistic effects on spacecraft materials MRS Bull. 35 41-7

[3] Zhou Y J, Lu G and Yang J L 2015 Finite element study of energy absorption foams for headgear in football (soccer) games Mater. Des. 88 162-9

[4] McAllister T W, Ford J C, Ji S, Beckwith J G, Flashman L a, Paulsen K and Greenwald R M 2012 Maximum principal strain and strain rate associated with concussion diagnosis correlates with changes in corpus callosum white matter indices. Ann. Biomed. Eng. 40 127-40

[5] Cao A, Dickrell P L, Sawyer W G, Ghasemi-Nejhad M N and Ajayan P M 2005 Super-compressible foamlike carbon nanotube films. Science 310 1307-10

[6] Thevamaran R, Meshot E R and Daraio C 2015 Shock formation and rate effects in impacted carbon nanotube foams Carbon 84 390-8

[7] Volder M De, Tawfick S, Baughman R and Hart A J 2013 Carbon nanotubes: Present and future commercial applications Science. 339 535-9

[8] Liu L, Ma W and Zhang Z 2011 Macroscopic carbon nanotube assemblies: Preparation, properties, and potential applications. Small 7 1504-20

[9] Deck C P, Flowers J, McKee G S B and Vecchio K 2007 Mechanical behavior of ultralong multiwalled carbon nanotube mats J. Appl. Phys. 10123512

[10] Yaglioglu O, Cao A, Hart A J, Martens R and Slocum A H 2012 Wide range control of microstructure and mechanical properties of carbon nanotube forests: A comparison between fixed and floating catalyst CVD techniques Adv. Funct. Mater. 22 5028-37

[11] Thevamaran R, Raney J R and Daraio C 2016 Rate-sensitive strain localization and impact response of carbon nanotube foams with microscale heterogeneous bands Carbon 101 184-90

[12] Raney J R, Misra A and Daraio C 2011 Tailoring the microstructure and 
[13] Suhr J, Victor P, Ci L, Sreekala S, Zhang X, Nalamasu O and Ajayan P M 2007

Fatigue resistance of aligned carbon nanotube arrays under cyclic compression. Nat. Nanotechnol. 2 417-21

[14] Bradford P D, Wang X, Zhao H and Zhu Y T 2011 Tuning the compressive mechanical properties of carbon nanotube foam Carbon. $492834-41$

[15] Lattanzi L, De Nardo L, Raney J R and Daraio C 2014 Geometry-induced mechanical properties of carbon nanotube foams $A d v$. Eng. Mater. 15 1-6

[16] Lattanzi L, Thevamaran R, De Nardo L and Daraio C 2015 Dynamic behavior of vertically aligned carbon nanotube foams with patterned microstructure $A d v$. Eng. Mater.

[17] Gui X, Wei J, Wang K, Cao A, Zhu H, Jia Y, Shu Q and Wu D 2010 Carbon nanotube sponges. Adv. Mater. 22 617-21

[18] Shan C, Zhao W, Lu L, O’Brien D J, Li Y, Cao Z, Elias A L, Terrones M, Wei B and Suhr J 2013 Three-Dimensionál Nitrogen-Doped Multiwall Carbon Nanotube Sponges with Tunable Properties. Nano Lett.

[19] Dai Z, Liu L, Qi X, Kuang J, Wei Y, Zhu H and Zhang Z 2016 Three-dimensional sponges with super mechanical stability: harnessing true elasticity of individual carbon nanotubes in macroscopic architectures Sci. Rep. 618930

[20] Luo S, Luo Y, Wu H, Li M, Yan L, Jiang K, Liu L, Li Q, Fan S and Wang J 2017 Self-assembly of 3D Carbon Nanotube Sponges: A Simple and Controllable Way to Build Macroscopic and Ultralight Porous Architectures Adv. Mater. 291603549

[21] Lin Z, Zeng Z, Gui X, Tang Z, Zou M and Cao A 2016 Carbon Nanotube Sponges, Aerogels, and Hierarchical Composites: Synthesis, Properties, and Energy Applications Adv. Energy Mater. 6 1-26

[22] Gui X, Li H, Wang K, Wei J, Jia Y, Li Z, Fan L, Cao A, Zhu H and Wu D 2011 Recyclable carbon nanotube sponges for oil absorption Acta Mater. 59 4798-804

[23] Karakaya M, Saini D, Podila R, Skove M J, Rao A M, Thevamaran R and Daraio C 2014 Self-Assembled Recyclable Hierarchical Bucky Aerogels Adv. Eng. Mater.

[24] Li H, Gui X, Zhang L, Wang S, Ji C, Wei J, Wang K, Zhu H, Wu D and Cao A 2010 Carbon nanotube sponge filters for trapping nanoparticles and dye molecules from water Chem. Commun. $\mathbf{4 6} 7966-8$

[25] Gui X, Zeng Z, Cao A, Lin Z, Zeng H, Xiang R, Wu T, Zhu Y and Tang Z 2012 Elastic shape recovery of carbon nanotube sponges in liquid oil J. Mater. Chem. 22 $18300-5$ 
[26] Zeng Z, Gui X, Gan Q, Lin Z, Zhu Y, Zhang W, Xiang R, Cao A and Tang Z 2014 Integrated random-aligned carbon nanotube layers: deformation mechanism under compression. Nanoscale 6 1748-55

[27] Xu M, Futaba D N, Yamada T, Yumura M and Hata K 2010 Carbon nanotubes with temperature-invariant viscoelasticity from -196 degrees to 1000 degrees C. Science 330 1364-8

[28] Liu Q, Li M, Gu Y, Wang S, Zhang Y and Li Q 2015 Interlocked CNT nétworks with high damping and storage modulus Carbon. 86 46-53

[29] Chen H, Zhang L, Chen J, Becton M, Wang X and Nie H2016 Energy dissipation capability and impact response of carbon nanotube buckypaper: A coarse-grained molecular dynamics study Carbon. 103 242-54

[30] Raney J, Wang R and Daraio C 2012 Control of microstructural heterogeneities in carbon nanotube foams Carbon. 52 193-200

[31] Lin Z, Gui X, Zeng Z, Liang B, Chen W, Liu M, Zhu Y, Cao A and Tang Z 2015 Biomimetic Carbon Nanotube Films with Gradient Structure and Locally Tunable Mechanical Property Adv. Funct. Mater. $257173 \rightarrow 9$

[32] Kim K H, Oh Y and Islam M F 2012 Graphene coating makes carbon nanotube aerogels superelastic and resistant to fatigue. Nat. Nanotechnol. 7 562-6

[33] Zhao W, Li Y, Wang S, He X, Shang Y, Peng Q, Wang C, Du S, Gui X, Yang Y, Yuan Q, Shi E, Wu S, Xu W and Cao A 2014 Elastic improvement of carbon nanotube sponges by depositing amorphous carbon coating Carbon. 76 19-26

[34] Kumar A, Maschmann M R, Hodson S L, Baur J and Fisher T S 2015 Carbon nanotube arrays decorated with multi-layer graphene-nanopetals enhance mechanical strength and durability Carbon. 84 236-45

[35] Thevamaran R and Daraio C 2014 An experimental technique for the dynamic characterization of soft complex materials Exp. Mech. 54 1319-28

[36] Gibson L and Ashby M 1999 Cellular solids (Cambridge University Press)

[37] Thevamaran R, Karakaya M, Meshot E R, Fischer A, Podila R, Rao A M and Daraio C 2015 Anomalous impact and strain responses in helical carbon nanotube foams RSGAdv. 529306-11 